\title{
Intereleukin-10 Promoter Polymorphism in Mild Cognitive Impairment and in Its Clinical Evolution
}

\author{
Beatrice Arosio, ${ }^{1}$ Luigina Mastronardi, ${ }^{1}$ Carlo Vergani, ${ }^{1}$ and Giorgio Annoni ${ }^{2}$ \\ ${ }^{1}$ Department of Internal Medicine, Università degli Studi di Milano, Geriatric Unit, \\ Fondazione IRCCS Cà Granda Ospedale Maggiore Policlinico, Via Pace 9, 20122 Milano, Italy \\ ${ }^{2}$ Department of Internal Medicine and Prevention, Università degli Studi di Milano-Bicocca, \\ Geriatric Clinic, San Gerardo Hospital, Via Pergolesi 33, 20052 Monza, Italy \\ Correspondence should be addressed to Giorgio Annoni, giorgio.annoni@unimib.it
}

Received 1 April 2010; Accepted 17 June 2010

Academic Editor: Diana Paleacu

Copyright (๑) 2010 Beatrice Arosio et al. This is an open access article distributed under the Creative Commons Attribution License, which permits unrestricted use, distribution, and reproduction in any medium, provided the original work is properly cited.

Specific proinflammatory alleles are associated with higher risk of Alzheimer disease (AD) in different onset age. The homozygosis for the A allele of - 1082 polymorphism (G/A) of interleukin-10 (IL-10) promotes a higher risk of AD and reduced IL-10 generation in peripheral cells after amyloid stimulation. In this paper we analysed genotype and allele frequencies of this polymorphism in 138 subjects with mild cognitive impairment (MCI) diagnosed, respectively, as amnestic (a-MCI) and multiple impaired cognitive domains (mcd-MCI). The genotype frequencies were similar in a-MCI and AD subjects, whereas in mcd-MCI comparable to controls (AA genotype: $50 \%$ in a-MCI, $49.2 \%$ in $\mathrm{AD}, 28.7 \%$ in mcd-MCI and $31.8 \%$ in controls). Consequently, both allele and genotype distributions were significantly different between a-MCI and mcd-MCI (allele: $P=.02$, genotype: $P<.05$ ). These results support the theory that polymorphisms of cytokine genes can affect neurodegeneration and its clinical progression. IL-10 may partly explain the conversion of a-MCI to AD or be a genetic marker of susceptibility.

\section{Introduction}

The pathogenic process of Alzheimer's disease (AD) starts decades before the clinical onset of the disease [1]. During this preclinical phase, there is a gradual loss of axons and neurons, and at a certain threshold the first symptoms, most often impaired episodic memory, appear. At this stage, patients do not fulfil the criteria for dementia and may be diagnosed with mild cognitive impairment (MCI). There is considerable clinical heterogeneity of this pathology since different clinical patterns can be recognized: amnestic MCI (a-MCI), MCI with multiple impaired cognitive domains (mcd-MCI), and single nonmemory domain MCI [2]. Although a-MCI may be the preclinical stage of $\mathrm{AD}$, there is no established method to predict progression to $\mathrm{AD}$ in individuals with MCI.

Inflammation is accepted to be a feature of $\mathrm{AD}[3,4]$ and the pathogeneses of neurodegeneration have been at least in part attributed to the release of proinflammatory cytokines from brain resident cells $[5,6]$ and, although less consistently, from peripheral cell $[7,8]$. Furthermore, an increased intrathecal production of the proinflammatory cytokine TNF- $\alpha$ and a decreased production of the antiinflammatory cytokine TGF- $\beta$ have been demonstrated in the brain of patients with $\mathrm{MCI}$, suggesting there is a proinflammatory state in such patients at high risk for $\mathrm{AD}$ [9].

Moreover, circulating acute phase reactant levels in middle age predict $\mathrm{AD}$ risk in old age and in particular certain functional promoter polymorphisms in cognate genes that modulate inflammation are often found at elevated frequency among $\mathrm{AD}$ cases.

Recently specific risk sets of proinflammatory alleles were identified that characterize $\mathrm{AD}$ in different onset age (before age 65 , at ages 65-74, and at older ages) [10].

These alleles comprise also the -1082 promoter gene polymorphisms of IL-10 (G/A substitution) [11].

IL-10 maps to chromosome 1 between $1 \mathrm{q} 31$ and $1 \mathrm{q} 32$ is highly polymorphic, and its production is correlated to biallelic polymorphisms at positions -1082 ( $G$ to A), 
-819 ( $\mathrm{T}$ to $\mathrm{C}$ ), and -592 (A to C). The polymorphism at position -1082 lies within an Ets (E-twenty-six specific)like recognition site and may affect the binding of this transcriptional factor and, therefore, alter transcription activation; the -1082 A allele correlates with low IL-10 generation after stimulation of $\mathrm{T}$ cells in vitro [12], while polymorphisms at positions -819 and -592 do not seem to be involved.

In a previous study, we found that the homozygosis for the A allele of the IL-10 - 1082 G/A single nucleotide polymorphism (SNP) was associated with six-fold higher risk of $\mathrm{AD}$. In the same study, we also analysed the production of IL10 in Peripheral Blood Mononuclear Cells (PBMCs) of AD patients and age-matched controls after specific stimulation with amyloid peptide, LPS, and Flu. Since the generation of IL-10 was reduced in patients after amyloid stimulation, we concluded that these specific immune responses may be selectively impaired in $\mathrm{AD}$ [13].

The aim of this study was to analyse the genotype and allele frequencies of these IL-10 SNPs in 138 subjects with $\mathrm{MCI}$ and to compare them with those previously shown in $\mathrm{AD}$ and healthy controls (HCs) [13].

\section{Materials and Methods}

2.1. Study Protocol. This study comprised 138 subjects with MCI age $80.37 \pm 5.93$ years (mean \pm standard deviation (SD)). All patients were Caucasian, living in Northern Italy, and selected from a larger ambulatory population sample followed at the Geriatric Unit of the Ospedale Maggiore Policlinico IRCCS, University of Milan, Italy and the Geriatric Clinic of the University of Milan-Bicocca, Italy.

At enrolment, MCI subjects were divided into two groups based on cognitive features and diagnosed, respectively, as aMCI (30 patients) and mcd-MCI (108 patients).

In particular, a-MCI met the criteria described by Petersen [14]: subjects with memory impairment only ( $>1.5 \mathrm{SD}$ above the age- and education-specific norms) and no difficulties in any other area of cognitive functions. mcd-MCIs were subjects diagnosed with impairment in at least two cognitive domains of more than 1SD below the mean of the respective age- and education-matched population, and with cognitive decline confirmed by the individuals themselves or reliable informants, but in whom no diagnosis of dementia could be achieved. A cut-off score of $1 \mathrm{SD}$ was applied, which is less severe than that used for a-MCI, in order to obtain higher diagnostic sensitivity even though diagnostic specificity was reduced. Because the presence of more than one cognitive deficit and frequently initial impairment in Lawton's instrumental activities of daily living also characterized mcd-MCI, it may be mistaken for dementia; thus, a less severe criterion ( $>1 \mathrm{SD}$ ) allows better differentiation between mcd-MCI and dementia [15].

At this time, 74 patients out of the 138 completed a four-year follow-up and 24 were diagnosed with AD, 22 with vascular dementia $(\mathrm{MCI} \rightarrow \mathrm{VD})$, and 28 with stable MCI [16]. Subjects who developed AD during follow-up were required to meet the DSM IV (Diagnostic and Statis- tical Manual of mental Disorders-4th ed.) and NINCDSADRDA (National Institute of Communicative Disorders and Stroke-Alzheimer's Disease and Related Disorders Association Work Group) criteria [17].

Within $\mathrm{MCI}$ who progressed to $\mathrm{AD}(\mathrm{MCI} \rightarrow \mathrm{AD})$, only two were diagnosed as mcd-MCI at enrolment; all the others were diagnosed as a-MCI.

In order to minimize the risk of possible inflammatory processes, all subjects were selected in the absence of clinical signs of inflammation (e.g., normal body temperature, no concomitant inflammatory condition) and with normal blood chemistry (red blood cell sedimentation rate, albumin, transferring, and $\mathrm{C}$ reactive protein plasma levels).

Informed consent was obtained from all subjects and the Ethics Committee of both universities approved the study, which was conducted according to the Helsinki II declaration. This population was matched with AD patients $(n=63)$ and nonsdemented sex- and age-matched healthy controls $(n=63)$ enrolled for our previous study [13].

2.2. Gene Polymorphism Analysis. Whole blood was collected by venipuncture in Vacutainer tubes containing EDTA (Becton Dickinson Co., Rutherford, NJ).

Genomic DNA was extracted by the salting-out method as described in [18]. The concentration and purity of DNA were determined by spectrophotometric analysis. In order to establish IL-10 genotypes we employed a polymerase chain reaction using sequence-specific primers (PCR-SSPs). The sequence in the promoter region of the IL-10 gene (polymorphic positions $-1082,-819$, and -592 ) was amplified using the cytokine genotyping tray method (One Lambda, Canoga Park, CA, USA). The human $\beta$-globin gene was amplified as an internal control for the genomic DNA preparation. PCR conditions were indicated by the One Lambda PCR program (OLI-1) and the PCR products were visualised by electrophoresis in $2.5 \%$ agarose gel.

ApoE genotypes were determined by means of PCR amplification of a 234 base-pair fragment of exon 4 of the ApoE gene, followed by digestion with Cfol. The restriction patterns were revealed by means of $4 \%$ agarose gel electrophoresis [13].

2.3. Statistical Analysis. Statistical analysis was performed with the SPSS statistical package (SPSS version 17, Chicago, IL). Genotype and allele frequencies in the study groups were compared using the $\chi^{2}$-test. $P<.05$ was taken as the cut-off for statistical significance.

\section{Results}

3.1. Distribution of IL-10 Genotypes in MCI Subjects. The genotype and allele frequencies of the biallelic polymorphism at position -1082 are reported in Table 1. This SNP alters transcriptional activation with a gene dosagerelated effect, so GG genotype correlates with high, GA with intermediate, and AA with low IL-10 production after stimulation of T cells in vitro [12]. 
TABle 1: Distribution of genotype and allele frequencies of -1082 (G/A) SNP in Alzheimer's disease patients (AD), control subjects (CT), and mild cognitive impairment patients (MCI).

\begin{tabular}{lccccc}
\hline & GG $(\mathrm{H})$ & GA $(\mathrm{M})$ & $\mathrm{AA}(\mathrm{L})$ & $\mathrm{G}$ & $\mathrm{A}$ \\
\hline $\mathrm{AD}$ & $4(6.4 \%)$ & $28(44.4 \%)$ & $31(49.2 \%)$ & $36(28.6 \%)$ & $90(71.4 \%)$ \\
$\mathrm{CT}$ & $14(22.2 \%)$ & $29(46 \%)$ & $20(31.8 \%)$ & $57(45.2 \%)$ & $69(54.8 \%)$ \\
$\mathrm{MCI}$ & $21(15.2 \%)$ & $71(51.4 \%)$ & $46(33.3 \%)$ & $113(40.9 \%)$ & $163(59.1 \%)$ \\
\hline
\end{tabular}

Genotype: $\chi^{2}$ 9.480, d.f. $4 ; P=.05$.

Allele: $\chi^{2} 8.257$, d.f. $2 ; P=.02$.

TABLE 2: Distribution of genotype and allele frequencies of -1082 (G/A) SNP in amnestic MCI (a-MCI) and multiple cognitive domains MCI patients (mcd-MCI).

\begin{tabular}{lccccc}
\hline & GG $(\mathrm{H})$ & GA $(\mathrm{M})$ & AA $(\mathrm{L})$ & $\mathrm{G}$ & $\mathrm{A}$ \\
\hline a-MCI & $1(3.3 \%)$ & $14(46.7 \%)$ & $15(50 \%)$ & $16(26.7 \%)$ & $44(73.3 \%)$ \\
mcd-MCI & $20(18.5 \%)$ & $57(52.8 \%)$ & $31(28.7 \%)$ & $97(44.9 \%)$ & $119(55.1 \%)$ \\
\hline
\end{tabular}

Genotype: $\chi^{2}$ 6.927, d.f. $2 ; P<.05$.

Allele: $\chi^{2} 5.729$, d.f. $1 ; P=.02$.

TABle 3: Distribution of genotype and allele frequencies of -1082 (G/A) SNP in MCIs that remain stable, progressed to AD (MCI $\rightarrow$ AD), and progressed to $\mathrm{VD}(\mathrm{MCI} \rightarrow \mathrm{VD})$.

\begin{tabular}{lccccc}
\hline & GG $(\mathrm{H})$ & GA $(\mathrm{M})$ & AA $(\mathrm{L})$ & $\mathrm{G}$ & $\mathrm{A}$ \\
\hline $\mathrm{MCI}$ stable & $8(28.6 \%)$ & $12(42.8 \%)$ & $8(28.6 \%)$ & $28(50 \%)$ & $28(50 \%)$ \\
$\mathrm{MCI} \rightarrow \mathrm{AD}$ & $2(8.3 \%)$ & $12(50 \%)$ & $10(41.7 \%)$ & $16(33.3 \%)$ & $32(66.7 \%)$ \\
$\mathrm{MCI} \rightarrow \mathrm{VD}$ & $5(22.7 \%)$ & $11(50 \%)$ & $6(27.3 \%)$ & $21(47.7 \%)$ & $23(52.3 \%)$ \\
\hline
\end{tabular}

Genotype distribution compared percentages: $\chi^{2} 15.604$, d.f. $4 ; P=.004$.

Allele distributions compared percentages: $\chi^{2} 6.661$, d.f. $2 ; P<.05$.

As previously described [13], AD patients show a significant higher frequency of the $-1082 \mathrm{~A}$ low producer allele, which skews the genotype distribution in $\mathrm{AD}$ compared to $\mathrm{HC}$, with a significant decrease of -1082 GG high producer genotype.

MCI subjects as a whole had an intermediate pattern between $\mathrm{AD}$ and $\mathrm{HC}$ subjects, the percentages of $\mathrm{G}$ allele and GG genotype being $40.9 \%$ and $15.2 \%$, while the percentage of A allele and AA genotype being 59.1\% and 33.3\%, respectively, (allele: $P=.02$, genotype: $P=.05$ ) (Table 1 ).

It is interesting to note that the genotype frequencies of the -1082 SNP in a-MCI subjects were similar to those of $\mathrm{AD}$ subjects, whereas those of mcd-MCI were comparable to $\mathrm{HC}$ (AA genotype $50 \%$ in a-MCI and $49.2 \%$ in $\mathrm{AD}$; $28.7 \%$ and $31.8 \%$ in mcd-MCI and HC, resp.) (Table 2). Consequently, the allele and genotype distributions were significantly different between a-MCI and mcd-MCI (allele: $P=.02$, genotype: $P<.05$ ).

The same SNP is linked with two other SNPs at positions -819 and -592 . They combine with microsatellite alleles to form haplotypes where the difference in IL-10 production is mainly accounted by the $-1082 \operatorname{SNP}[19,20]$. The genotype and allele frequencies of -819 and -592 SNPs were distributed similarly in our samples (data not shown).

3.2. Distribution of Apolipoprotein E Genotype in MCI Subjects. The frequency of ApoE $\varepsilon 4$ in our sample was in line with the data already published [21-24]. In particular genotyping of our MCI patients globally considered revealed the presence of $\varepsilon 4$ allele in $40 \%$ of cases and, during followup, in $54 \%$ of MCI $\rightarrow \mathrm{AD}$ and $39 \%$ in stable MCI. The ApoE4 status is an independent risk factor for $\mathrm{AD}$ [13].

3.3. Follow-Up. After a 4-year follow-up $24 \mathrm{MCI}$ progressed to $\mathrm{AD}(\mathrm{MCI} \rightarrow \mathrm{AD})$ [16] and 22 progressed to vascular dementia $(\mathrm{MCI} \rightarrow \mathrm{VD})$. Table 3 shows -1082 SNP distributions in MCI progressing and not progressing to $\mathrm{AD}$ (stable MCI).

In $\mathrm{MCI} \rightarrow \mathrm{AD}$ both $\mathrm{A}$ allele and $\mathrm{AA}$ genotype were higher than in stable MCI and in MCI $\rightarrow$ VD.

Due to the limited number of patients that completed the follow-up period, the data reached the statistical significances only comparing genotype and allele percentage (allele: $P<.05$, genotype $P=.004)$.

\section{Discussion}

A "cytokine cycle" has been proposed where [25] the anti-inflammatory cytokines (IL-4, IL-10, and IL-13) regulate $\beta$-amyloid-induced microglial/macrophage inflammatory responses and modify the microglial activity surrounding amyloid neuritic plaques [26]. These cytokines can inhibit the induction of IL-1, TNF- $\alpha$, and MCP-1 in differentiated human monocytes and, above all, IL10 causes dose-dependent inhibition of the IL- 6 secretion induced by $\beta$-amyloid in these cells and in murine microglia [25]. 
In a previous paper, we described not only a significantly higher percentage of IL-10 - 1082 AA low-producing genotype among $\mathrm{AD}$ cases, but also a reduced IL-10 generation in peripheral blood mononuclear cells from these patients after $\beta$-amyloid stimulation [13].

Interestingly a report on Italian centenarians, who are clearly less prone than younger persons to age-related diseases, showed that extreme longevity is significantly associated with the high IL-10-producing genotypes [27].

In the present study, the allele frequencies of -1082 SNP in a-MCI subjects were similar to those of $\mathrm{AD}$ patients, whereas those of mcd-MCI were comparable to $\mathrm{HC}$ (the frequencies of the low-producer AA genotype were 50\% and $28.7 \%$, in a-MCI and mcd-MCI, resp.).

It is to note that, after an adequate period of followup, the twenty-four a-MCI subjects that progressed to AD showed a higher percentage of AA carriers (41.7\%) compared to those of MCI that remain stable (28.6\%) and compared to those progressed in vascular dementia (27.3\%). The similar genotype distribution of this IL-10 SNP in AD and a-MCI but not in mcd-MCI and the data retrospectively obtained after the follow-up suggest that it is potentially involved in the conversion of a-MCI to AD.

However, our results support the theory that the overall risk of developing $\mathrm{AD}$ may be governed by a multifactorial "susceptibility profile" and that polymorphisms of cytokine genes can affect neurodegeneration and its clinical progression.

In addiction, IL-10 may partly explain the conversion of a-MCI to AD or, at least, be a genetic marker of susceptibility [28].

Therefore, it is extremely relevant to closely define intrinsic (i.e., genetic) individual risk profiles in prevention and treatment trials. The finding that the set of gene variants in innate immunity associated with earlier onset predicted rapid clinical progression suggests that interventions to control inflammation might be useful especially for relatively younger cases to delay disease progression.

\section{References}

[1] J. L. Price and J. C. Morris, "Tangles and plaques in nondemented aging and "preclinical" Alzheimer's disease," Annals of Neurology, vol. 45, no. 3, pp. 358-368, 1999.

[2] R. C. Petersen, R. Doody, A. Kurz et al., "Current concepts in mild cognitive impairment," Archives of Neurology, vol. 58, no. 12, pp. 1985-1992, 2001.

[3] H. W. Querfurth and F. M. LaFerla, "Alzheimer's disease," The New England Journal of Medicine, vol. 362, no. 4, pp. 329-344, 2010.

[4] T. Wyss-Coray, "Inflammation in Alzheimer disease: driving force, bystander or beneficial response?" Nature Medicine, vol. 12, no. 9, pp. 1005-1015, 2006.

[5] E. Tarkowski, K. Blennow, A. Wallin, and A. Tarkowski, "Intracerebral production of tumor necrosis factor- $\alpha$, a local neuroprotective agent, in Alzheimer disease and vascular dementia," Journal of Clinical Immunology, vol. 19, no. 4, pp. 223-230, 1999.

[6] E. Tarkowski, A.-M. Liljeroth, Å. Nilsson, L. Minthon, and K. Blennow, "Decreased levels of intrathecal interleukin 1 receptor antagonist in Alzheimer's disease," Dementia and Geriatric Cognitive Disorders, vol. 12, no. 5, pp. 314-317, 2001.

[7] P. Bermejo, S. Martín-Aragón, J. Benedí et al., "Differences of peripheral inflammatory markers between mild cognitive impairment and Alzheimer's disease," Immunology Letters, vol. 117, no. 2, pp. 198-202, 2008.

[8] K. Bonotis, E. Krikki, V. Holeva, C. Aggouridaki, V. Costa, and S. Baloyannis, "Systemic immune aberrations in Alzheimer's disease patients," Journal of Neuroimmunology, vol. 193, no. 12, pp. 183-187, 2008.

[9] S. Ray, M. Britschgi, C. Herbert et al., "Classification and prediction of clinical Alzheimer's diagnosis based on plasma signaling proteins," Nature Medicine, vol. 13, no. 11, pp. 13591362, 2007.

[10] F. Licastro, E. Porcellini, C. Caruso, D. Lio, and E. H. Corder, "Genetic risk profiles for Alzheimer's disease: integration of APOE genotype and variants that up-regulate inflammation," Neurobiology of Aging, vol. 28, no. 11, pp. 1637-1643, 2007.

[11] D. Lio, F. Licastro, L. Scola et al., "Interleukin-10 promoter polymorphism in sporadic Alzheimer's disease," Genes and Immunity, vol. 4, no. 3, pp. 234-238, 2003.

[12] J. M. Kim, C. I. Brannan, N. G. Copeland, N. A. Jenkins, T. A. Khan, and K. W. Moore, "Structure of the mouse IL-10 gene and chromosomal localization of the mouse and human genes," Journal of Immunology, vol. 148, no. 11, pp. 3618-3623, 1992.

[13] B. Arosio, D. Trabattoni, L. Galimberti et al., "Interleukin10 and interleukin- 6 gene polymorphisms as risk factors for Alzheimer's disease," Neurobiology of Aging, vol. 25, no. 8, pp. 1009-1015, 2004.

[14] R. C. Petersen, "Mild cognitive impairment as a diagnostic entity," Journal of Internal Medicine, vol. 256, no. 3, pp. 183194, 2004.

[15] M. Zanetti, C. Ballabio, C. Abbate, C. Cutaia, C. Vergani, and L. Bergamaschini, "Mild cognitive impairment subtypes and vascular dementia in community-dwelling elderly people: a 3-year follow-up study," Journal of the American Geriatrics Society, vol. 54, no. 4, pp. 580-586, 2006.

[16] O. Hansson, H. Zetterberg, P. Buchhave, E. Londos, K. Blennow, and L. Minthon, "Association between CSF biomarkers and incipient Alzheimer's disease in patients with mild cognitive impairment: a follow-up study," Lancet Neurology, vol. 5, no. 3, pp. 228-234, 2006.

[17] G. McKhann, D. Drachman, and M. Folstein, "Clinical diagnosis of Alzheimer's disease: report of the NINCDS-ADRDA work group under the auspices of Department of Health and Human Services Task Force on Alzheimer's disease," Neurology, vol. 34, no. 7, pp. 939-944, 1984.

[18] S. A. Miller, D. D. Dykes, and H. F. Polesky, "A simple salting out procedure for extracting DNA from human nucleated cells," Nucleic Acids Research, vol. 16, no. 3, p. 1215, 1988.

[19] J. Eskdale, P. Wordsworth, S. Bowman, M. Field, and G. Gallagher, "Association between polymorphisms at the human IL10 locus and systemic lupus erythematosus," Tissue Antigens, vol. 49, no. 6, pp. 635-639, 1997.

[20] J. Eskdale, G. Gallagher, C. L. Verweij, V. Keijsers, R. G. J. Westendorp, and T. W. J. Huizinga, "Interleukin 10 secretion in relation to human IL-10 locus haplotypes," Proceedings of the National Academy of Sciences of the United States of America, vol. 95, no. 16, pp. 9465-9470, 1998.

[21] D. J. Berlau, M. M. Corrada, E. Head, and C. H. Kawas, "ApoE $\varepsilon 2$ is associated with intact cognition but increased Alzheimer pathology in the oldest old," Neurology, vol. 72, no. 9, pp. 829$834,2009$. 
[22] G. Bu, "Apolipoprotein E and its receptors in Alzheimer's disease: pathways, pathogenesis and therapy," Nature Reviews Neuroscience, vol. 10, no. 5, pp. 333-344, 2009.

[23] P. P. Singh, M. Singh, and S. S. Mastana, "APOE distribution in world populations with new data from India and the UK," Annals of Human Biology, vol. 33, no. 3, pp. 279-308, 2006.

[24] J. Kim, J. M. Basak, and D. M. Holtzman, "The role of apolipoprotein E in Alzheimer's disease," Neuron, vol. 63, no. 3, pp. 287-303, 2009.

[25] A. M. Szczepanik, S. Funes, W. Petko, and G. E. Ringheim, "IL-4, IL-10 and IL-13 modulate A $\beta$ (1-42)-induced cytokine and chemokine production in primary murine microglia and a human monocyte cell line," Journal of Neuroimmunology, vol. 113, no. 1, pp. 49-62, 2001.

[26] C. C. Chao, T. W. Molitor, and S. Hu, "Neuroprotective role of IL-4 against activated microglia," Journal of Immunology, vol. 151, no. 3, pp. 1473-1481, 1993.

[27] D. Lio, L. Scola, A. Crivello et al., "Gender-specific association between-1082 IL-10 promoter polymorphism and longevity," Genes and Immunity, vol. 3, no. 1, pp. 30-33, 2002.

[28] O. Combarros, P. Sánchez-Juan, J. A. Riancho et al., "Aromatase and interleukin-10 genetic variants interactively modulate Alzheimer's disease risk," Journal of Neural Transmission, vol. 115, no. 6, pp. 863-867, 2008. 